\title{
Many cytokines are very useful therapeutic targets in disease
}

\author{
Marc Feldmann \\ Kennedy Institute of Rheumatology, Faculty of Medicine, Imperial College London, London, United Kingdom.
}

\begin{abstract}
Cytokines are a large family of more than 100 small proteins that function as short-range mediators involved in essentially all biological processes. They have been found to be important rate-limiting signals, and it is now known that blocking some cytokines, e.g., TNF- $\alpha$, and cytokine receptors, such as human EGFR 1 (HER1) or HER2, yields effective therapeutics that address unmet needs. This Review Series surveys three chronic inflammatory disease areas and two forms of cancer and discusses the important role of cytokines and their receptors in these disease processes. Their role as potential therapeutic targets is also highlighted.
\end{abstract}

Cytokines, low-MW protein mediators that usually act at short range between neighboring cells, have been studied extensively for the past 30 years. These molecules, previously also termed interleukins, interferons, growth factors, and TNFs, among other designations, are involved in essentially every important biological process, from cell proliferation to inflammation, immunity, migration, fibrosis, repair, and angiogenesis $(1,2)$. As these molecules and their associated receptors provide key signals for important processes, it is not surprising that abnormalities in cytokines, their receptors, and the signaling pathways that they initiate are involved in a wide variety of diseases. Indeed, they have a role in far too many diseases for each to be covered in this Review Series. Rather, representative examples of the cytokines involved in some forms of cancer and immune and inflammatory diseases are discussed. The critical issue in each case is the importance of cytokines as therapeutic targets, and the overwhelming message is that "anti-cytokine medicine" is a rapidly growing field with major pharmaceutical impact.

\section{Cytokines: from identification to the clinic}

The field of cytokines came of age in the late 1970s with the introduction of molecular biological approaches that resulted first in the cloning of IFNs, initially IFN- $\beta$ by Tada Taniguchi (3) and IFN- $\alpha$ by both Charles Weissman's group and David Goeddel's colleagues (4, 5). By the mid-1980s, there was a plethora of well-defined cytokines and cytokine receptors that could be unambiguously studied, using molecular tools, such as cDNA probes, and antibodies that had been produced to recognize the pure recombinant proteins. All this was a long way from the 1960s and 1970s, when all researchers had were many uncharacterized bioactivities in cell supernatants termed simply by activity, e.g., lymphocyte-activating factor (6), macrophageactivating factor (7), and leukocyte pyrogen (8).

All the tools available by the mid-1980s enabled researchers to assess the expression of cytokines in physiologic and patho-

Nonstandard abbreviations used: $\mathrm{AD}$, Alzheimer disease; $\mathrm{CML}$, chronic myelogenous leukemia; COPD, chronic obstructive pulmonary disease; HER, human EGFR.

Conflict of interest: The author has multiple patents on aspects of anti-TNF therapy, has shares in Johnson \& Johnson and Schering-Plough, has received income from Roche, Celgene, Wyeth, Nuon, and Novo Nordisk, grants from Novo Nordisk and Wyeth, and received consulting/scientific advisory board payments from Roche, Celgene, Wyeth, Nuon, Novo Nordisk, Hydra Biosciences, Receptor BioLogix, Kahr, Vascular Biogenics, MaimoniDex, Teva, StormBio, and Trillium Therapeutics. The author is also a scientific advisor on grants to colleagues from Celgene, Receptor BioLogix, and Nuon.

Citation for this article: J. Clin. Invest. 118:3533-3536 (2008). doi:10.1172/JCI37346. logic states. The upregulated expression of cytokines in many different disease states led to an investigation of their role in the pathogenesis of disease, and the articles in this Review Series barely scratch the surface of the plethora of information now available. As cytokines are potent rate-limiting extracellular molecules, they are excellent targets for the products of the biotechnology industry, namely monoclonal antibodies and antibody-like receptor:Fc fusion proteins. These form the most specific therapeutics, more specific than small molecule organic chemicals, due to the greater surface of interaction of receptors and antibodies with their targets.

\section{Cytokines in immune and inflammatory disorders}

RA is a common autoimmune disease, with approximately $1 \%$ prevalence in the industrialized world (9), and cytokine expression in this disease has been extensively analyzed, an effort helped considerably by the accessibility of the diseased tissue (10). The first big success of anti-cytokine therapy, in the form of TNF- $\alpha$ blockade, was demonstrated in this disease, and it has now been repeatedly shown that blocking this single cytokine has marked beneficial effects on all aspects of disease activity and can prevent further joint destruction $(11,12)$. In addition, it has been determined that several other important chronic diseases respond to TNF- $\alpha$ blockade. In the first article in this Review Series, Fionula Brennan and Iain McInnes summarize the work leading to the routine clinical use of TNF- $\alpha$ blockade as a therapy for RA (13) and go on to review the extensive data on the function of many other cytokines in this disease. Due to the ease of performing clinical trials with well-established protocols, multiple cytokine blockade clinical trials have been performed in severe RA (e.g., ref. 14).

These clinical trials have had variable success, and it is not understood why in this disease, as in many others, there are differences between results in animal models, where many anti-cytokine therapies are very effective, and the human disease, treatment of which is proving more challenging. For example, in the mouse model of RA, blockade of IL- 1 is just as beneficial as TNF- $\alpha$ blockade, sometimes more so (15); but while IL-1 blockade in the form of IL-1 receptor antagonist (IL-1Ra) is effective and approved in RA (16), it is a less potent therapeutic approach than TNF- $\alpha$ blockade and hence has not been as extensively used in the clinic. Further, more potent, recently tested IL-1 inhibitors, such as a monoclonal antibody specific for IL-1 $\beta$ and an IL-1 receptor:Fc fusion protein, are no more effective in RA than is IL-1Ra. Very effective in clinical trials is an 
antibody specific for the IL-6R component of the receptor for IL-6, which is almost certain to be approved for clinical use (17). Proofof-principle studies have shown efficacy for a fusion protein that blocks two cytokines of the TNF superfamily, lymphotoxin- $\beta$ (LT- $\beta$ ) and ligand for herpesvirus entry mediator (LIGHT) (18), and for IL-15 blockade (19) but little efficacy for an antibody specific for the TNF superfamily member B lymphocyte stimulator (BLyS). Not yet known is the effect of blockade of many other cytokines that might have an important role in the pathogenesis of RA, including IL-12/23, IL-17, IL-18, IL-27, and IL-32.

In the second article in this Review Series, Peter Barnes provides an overview of the role of cytokines in two major pulmonary diseases: asthma and chronic obstructive pulmonary disease (COPD) (20). In these diseases, despite extensive analysis, little clinical success has been reported with therapeutics targeting cytokines. Why is this the case? As in RA, a large number of cytokines are upregulated, but therapeutics blocking the initial favorite, IL-5, did not succeed in clinical trials in asthma (21), nor did therapeutics blocking IL-4 (22). There is still optimism, however, that blocking IL-13 or IL-9 or blocking the IL-4/13 receptor may be beneficial in individuals with asthma. As asthma is considered a Th2 disease, it is of interest that blockade of TNF- $\alpha$, usually considered a Th1 cytokine, was successful in individuals with very severe asthma; hence, trials of TNF- $\alpha$ blockade are ongoing in this clinical setting. In COPD, short-term clinical trials ( 6 months) of TNF- $\alpha$ blockade showed no clinical benefit, and it is strange that such a shortterm trial was even attempted given the known slow progress of COPD, where two years of treatment is assumed to be necessary (23). In light of the lack of clinical success of therapeutics targeting cytokines in COPD or asthma, Barnes makes the interesting suggestion that a broader spectrum of antiinflammatory effects is needed to treat asthma and COPD than can be achieved simply by blocking any one specific cytokine or receptor. In RA, anti-TNF- $\alpha$ therapy induces a rapid reduction in other "downstream" inflammatory mediators, such as IL-1 and IL-6 (12), but it is conceivable that this does not happen in the lung, which has immunology and inflammatory constraints different from those of joints, as overexuberant inflammation in the lung is lethal.

In his Review, Lawrence Steinman discusses three important instances where cytokines have a major impact on CNS function: fever, MS, and Alzheimer disease (AD) (24). MS is an important neurodegenerative disease in which inflammation and cytokines are prominent in the affected tissue, and another cytokine, IFN- $\beta$, is often used for therapy (25). Clinical evidence that cytokines are involved in the pathogenesis of MS came initially from a clinical trial in which administration of IFN- $\gamma$ made the disease worse (26), establishing a presumptive pathogenic role for a major source of IFN- $\gamma, \mathrm{CD}^{+}$Th 1 cells. Subsequent analysis of MS lesions has revealed the expression of multiple cytokines and their downstream signaling molecules; IL-6, IL-17, IFN- $\gamma$, and osteopontin were prominent (27), but we await the results of clinical studies in which the therapeutic effects of blockade of these molecules are assessed.

Surprisingly, the effects of TNF- $\alpha$ blockade, which is beneficial in a number of diseases (including RA, ankylosing spondylitis, psoriasis, and inflammatory bowel disease), have been assessed in individuals with MS and were found to worsen the disease, augmenting relapse frequency (28). Reasons for this are likely to include the fact that TNF- $\alpha$ has neuroprotective effects and also that the chronic presence of TNF- $\alpha$ downregulates IL-12 and $\mathrm{T}$ cell responses, which are thus augmented in the periphery after TNF- $\alpha$ blockade. Problems (renal toxicity effects) were also encountered in a small pilot human trial to test the efficacy of administering TGF- $\beta$, expression of which is elevated in the brains of individuals with MS (29).

In contrast to the unsuccessful attempts to block TNF- $\alpha$ and administer TGF- $\beta$, administration of IFN- $\beta$ has a beneficial effect on individuals with MS. However, as Steinman discusses, the mechanism(s) underlying these beneficial effects of IFN- $\beta$ are not clear. There is evidence for downmodulation of the proinflammatory Th1 and Th17 pathways, diminution of HLA class II expression, reduction in IFN- $\gamma$ expression, reduction in MMP-2 and MMP-7 expression, reduction in IL-17 expression, and an increase in TNF- $\alpha$ levels (30-32). But which of these really matter is not yet known.

Of potential clinical importance in the pathogenesis of $\mathrm{AD}$ is the role of inflammation and cytokines (33). TGF- $\beta$ overexpression in mice engineered to develop AD reduced the plaque burden (34). Paradoxically, however, reducing TGF- $\beta$ signaling also reduced IL-6 expression and inflammation as well as plaque burden in other models of $\mathrm{AD}$, suggesting that the role of TGF- $\beta$ needs further evaluation (35). Thus, cytokines are intimately involved in several major neuropathologic states. Which cytokine-directed approaches are going to be therapeutically optimal remain to be ascertained.

\section{Cytokines in cancer}

In reviewing the role of cytokines in leukemogenesis, Kendall Smith and James Griffin explore chronologically the role of cytokines and cytokine receptors in the control of leukemic cell growth (36). During the 1960s, 1970s, and 1980s, there was an interplay among fields with no apparent initial connections, including cancer biology and normal immunology, from which emerged assays for $\mathrm{T}$ cell growth factors (37), the purification of protein with growth factor activity, the identification of its receptor (38), and the production of an antibody specific for the receptor (39).

These tools, along with many others generated at the same time, helped provide a new framework for lymphocyte growth, not as a direct response to antigenic stimuli, but as a response to antigen-induced growth factor release (40). The signaling pathways involved in cytokine responses have also been uncovered, and the work on tyrosine kinases, in particular the identification of the JAK family, and on IFN signaling, which established the importance of the STAT family of transcription factors, revealed major aspects of cytokine signaling.

With knowledge of the normal pathway of cell growth, the abnormalities in leukemia are easier to understand. The hallmark translocation of chronic myelogenous leukemia (CML), the Philadelphia chromosome, engenders a translocation of the breakpoint cluster region gene $(B C R)$ into the gene that encodes the ABL tyrosine kinase, leading to the synthesis of a $210-\mathrm{kDa} B C R-A B L$ protein with constitutive enzyme activity (41). This is the target of imatinib (Gleevec), which has revolutionized CML therapy (42) and stimulated much research into kinase inhibitors as therapeutic agents. The thrust of the Review by Smith and Griffin, which uses CML as an example, is that understanding the normal cytokine control of cell growth is the key to identifying other molecules involved in normal growth control and that this provides the basis for understanding the abnormalities in various forms of cancer and leukemia. From there flow multiple therapeutic opportunities.

A notable success of cancer research has been the unraveling of the HER family and the signaling pathways that they initiate 
to yield therapeutics for several major cancers, including cancers of the breast and colon. The current therapeutics target EGFR (also known as HER1) and HER2. In the final article in the Review Series, Michael Shepard and colleagues summarize the work since the 1980s leading to the clinical use of an antagonistic monoclonal antibody targeting HER2 for the treatment of cancer (43). Briefly, one of the key observations, first documented in Shepard's laboratory, was that nearly all laboratory cancer cell lines are resistant to TNF- $\alpha$-induced cytotoxicity (44). Further, cancer cell resistance to the cytotoxic effects of TNF- $\alpha$ could be induced by ligands for EGFR (which can form a heterodimer with HER2) and by overexpression of HER2 (45) as well as other oncogenes. These observations suggested that antagonizing HER2 should be therapeutic and initiated the search for antagonistic HER2-specific monoclonal antibodies, which culminated in the development of trastuzumab (Herceptin), which is now used routinely for the treatment of some aggressive forms of breast cancer (46).

In the Review, Shepard and colleagues argue that the success of monoclonal antibodies targeting HER family members in cancer lies in their capacity to disrupt the ability of these receptors to act as integrators of signals deriving from a multitude of other molecules, including GPCRs, TLRs, hormone receptors, and other cytokine receptors. Specifically, activation of GPCRs, such as the angiotensin receptor and chemokine receptors, can lead to MMP/ADAM-catalyzed release of ligands for HER family members, which can then activate their cognate receptors on cancer or stromal cells; this process is known as transactivation. Activation of TLRs, TNF receptors, and TGF- $\beta$ receptors also can result in the release of ligands for HER family members. The ability of HER family members to integrate these signals from these pathways can enable feedback loops to be generated that augment stromatumor cell interactions.
Although trastuzumab and the EGFR-specific monoclonal antibody cetuximab (Erbitux) are successful therapeutics, there are many patients who do not respond to these therapeutics, and resistance to them develops in many who do initially respond. Interestingly, it has been found that resistance to cetuximab can be reversed with an HER2-specific antibody (2C4) that interferes with HER2-EGFR heterodimerization. Furthermore, resistance to trastuzumab is associated with increased expression of EGFR and hence the presence of more EGFR-HER2 heterodimers. This raises the hypothesis that cooperation among EGFR family members is important in acquired resistance to therapeutics targeting these molecules. Shepard and colleagues thus suggest that targeting the HER family as a whole, or multiple members, might be of great therapeutic value.

\section{Future directions}

Although this Review Series skims the surface of the role of cytokines in disease processes, its goal is to trigger greater appreciation of the present therapeutic capacity of cytokines and cytokine receptors as well as their future potential. Cytokines are involved in essentially every major biological process, from inflammation to immune responses, cell growth, cell migration, fibrosis, and angiogenesis, so it is likely that every disease will involve multiple cytokines. Existing work documents that cytokines are potent ratelimiting signals and that some anti-cytokine therapeutics have had major clinical success. Moving forward, there is a lot more to be done, but I believe that clinical successes are inevitable.

Address correspondence to: Marc Feldmann, Kennedy Institute of Rheumatology Division, Imperial College Faculty of Medicine, 65 Aspenlea Road, London W6 8LH, United Kingdom. Phone: 44-20-8383-4400; Fax: 44-20-8563-0399; E-mail: m.feldmann@ imperial.ac.uk.
1. Vilcek, J., and Feldmann, M. 2004. Historical review: cytokines as therapeutics and targets of therapeutics. Trends Pharmacol. Sci. 25:201-209.

2. Oppenheim, J.J. 2001. Cytokines: past, present, and future. Int. J. Hematol. 74:3-8.

3. Taniguchi, T., Fujii-Kuriyama, Y., and Muramatsu, M. 1980. Molecular cloning of human interferon cDNA. Proc. Natl. Acad. Sci. U. S. A. 77:4003-4006.

4. Nagata, S., et al. 1980. Synthesis in E. coli of a polypeptide with human leukocyte interferon activity. Nature. 284:316-320.

5. Goeddel, D.V., et al. 1980. Synthesis of human fibroblast interferon by E. coli. Nucleic Acids Res. 8:4057-4074.

6. Gery, I., Gershon, R.K., and Waksman, B.H. 1972. Potentiation of the T-lymphocyte response to mitogens. I. The responding cell. J. Exp. Med. 136:128-142.

7. Hibbs, J.B., Jr., Taintor, R.R., Chapman, H.A., Jr., and Weinberg, J.B. 1977. Macrophage tumor killing: influence of the local environment. Science. 197:279-282.

8. Dinarello, C.A., Goldin, N.P., and Wolff, S.M. 1974. Demonstration and characterisation of two distinct human leukocyte pyrogens. J. Exp. Med. 139:1369-1381.

9. Lawrence, J.S. 1961. Prevalence of rheumatoid arthritis. Ann. Rheum. Dis. 20:11-17.

10. Feldmann, M., Brennan, F.M., and Maini, R.N. 1996. Role of cytokines in rheumatoid arthritis. Annu. Rev. Immunol. 14:397-440.

11. Elliott, M.J., et al. 1993. Treatment of rheumatoid arthritis with chimeric monoclonal antibodies to tumor necrosis factor alpha. Arthritis Rheum. 36:1681-1690.
12. Feldmann, M., and Maini, R.N. 2001. Anti-TNF alpha therapy of rheumatoid arthritis: what have we learned? Annu. Rev. Immunol. 19:163-196.

13. Brennan, F.M., and McInnes, I.B. 2008. Evidence that cytokines play a role in rheumatoid arthritis. J. Clin. Invest. 118:3537-3545.

14. Klareskog, L., et al. 2004. Therapeutic effect of the combination of etanercept and methotrexate compared with each treatment alone in patients with rheumatoid arthritis: double-blind randomised controlled trial. Lancet. 363:675-681.

15. Joosten, L.A., et al. 1999. IL-1 alpha beta blockade prevents cartilage and bone destruction in murine type II collagen-induced arthritis, whereas TNFalpha blockade only ameliorates joint inflammation. J. Immunol. 163:5049-5055.

16. Furst, D.E., et al. 2005. Updated consensus statement on biological agents, specifically tumour necrosis factor $\{$ alpha\} (TNF $\{$ alpha\}) blocking agents and interleukin-1 receptor antagonist (IL$1 \mathrm{ra})$, for the treatment of rheumatic diseases, 2005. Ann. Rheum. Dis. 64(Suppl. 4):iv2-iv14.

17. Nishimoto, N., et al. 2004. Treatment of rheumatoid arthritis with humanized anti-interleukin- 6 receptor antibody: a multicenter, double-blind, placebocontrolled trial. Arthritis Rheum. 50:1761-1769.

18. Baldassare, A., et al. 2007. Preliminary safety and efficacy of LT $\beta$ R-Ig (BG9924) in the treatment of rheumatoid arthritis (RA). Presented at the American College of Rheumatology Annual Scientific Meeting. November 6-11. Boston, Massachusetts, USA. Presentation no. 947.

19. Baslund, B., et al. 2005. Targeting interleukin-15 in patients with rheumatoid arthritis: a proof-ofconcept study. Arthritis Rheum. 52:2686-2692.
20. Barnes, P.J. 2008. The cytokine network in asthma and chronic obstructive pulmonary disease. J. Clin. Invest. 118:3546-3556.

21. Flood-Page, P., et al. 2007. A study to evaluate safety and efficacy of mepolizumab in patients with moderate persistent asthma. Am.J. Respir. Crit. Care Med. 176:1062-1071.

22. Borish, L.C., et al. 2001. Efficacy of soluble IL-4 receptor for the treatment of adults with asthma. J. Allergy Clin. Immunol. 107:963-970.

23. Rennard, S.I., et al. 2007. The safety and efficacy of infliximab in moderate to severe chronic obstructive pulmonary disease. Am. J. Respir. Crit. Care. Med. 175:926-934

24. Steinman, L. 2008. Nuanced roles of cytokines in three major human brain disorders. J. Clin. Invest. 118:3557-3563.

25. Arnason, B.G. 1999. Immunologic therapy of multiple sclerosis. Annu. Rev. Med. 50:291-302.

26. Panitch, H.S., Hirsch, R.L., Schindler, J., and Johnson, K.P. 1987. Treatment of multiple sclerosis with gamma interferon: exacerbations associated with activation of the immune system. Neurology. 37:1097-1102.

27. Chabas, D., et al. 2001. The influence of the proinflammatory cytokine, osteopontin, on autoimmune demyelinating disease. Science. 294:1731-1735.

28. The Lenercept Multiple Sclerosis Study Group and The University of British Columbia MS/MRI Analysis Group. 1999. TNF neutralization in MS: results of a randomized, placebo-controlled multicenter study. Neurology. 53:457-465.

29. Calabresi, P.A., et al. 1998. Phase 1 trial of transforming growth factor beta 2 in chronic progressive MS. Neurology. 51:289-292. 
30. Hall, G.L., Compston, A., and Scolding, N.J. 1997. Beta-interferon and multiple sclerosis. Trends Newrosci. 20:63-67.

31. Steinman, L. 2001. Multiple sclerosis: a two-stage disease. Nat. Immunol. 2:762-764.

32. Stuve, O., et al. 1996. Interferon beta-1b decreases the migration of Tlymphocytes in vitro: effects on matrix metalloproteinase-9. Ann. Neurol. 40:853-863.

33. Wyss-Coray, T. 2006. Inflammation in Alzheimer disease: driving force, bystander or beneficial response? Nat. Med. 12:1005-1015.

34. Wyss-Coray, T., et al. 2001. TGF-beta1 promotes microglial amyloid-beta clearance and reduces plaque burden in transgenic mice. Nat. Med. 7:612-618.

35. Town, T., et al. 2008. Blocking TGF-beta-Smad $2 / 3$ innate immune signaling mitigates Alzheimer-like pathology. Nat. Med. 14:681-687.

36. Smith, K.A., and Griffin, J.D. 2008. Following the cytokine signaling pathway to leukemogenesis: a chronology. J. Clin. Invest. 118:3564-3573.

37. Gillis, S., Ferm, M.M., Ou, W., and Smith, K.A. 1978. T cell growth factor: parameters of production and a quantitative microassay for activity. J. Immunol. 120:2027-2032.

38. Robb, R.J., Munck, A., and Smith, K.A. 1981. T cell growth factor receptors. Quantitation, specificity, and biological relevance. J. Exp. Med. 154:1455-1474.

39. Leonard, W.J., et al. 1982. A monoclonal antibody that appears to recognize the receptor for human T-cell growth factor; partial characterization of the receptor. Nature. 300:267-269.

40. Smith, K.A., Lachman, L.B., Oppenheim, J.J., and Favata, M.F. 1980. The functional relationship of the interleukins. J. Exp. Med. 151:1551-1556.

41. Ben-Neriah, Y., Daley, G.Q., Mes-Masson, A.M., Witte, O.N., and Baltimore, D. 1986. The chronic myelogenous leukemia-specific $\mathrm{P} 210$ protein is the product of the bcr/abl hybrid gene. Science. 233:212-214.
42. Druker, B.J., et al. 2001. Efficacy and safety of a specific inhibitor of the BCR-ABL tyrosine kinase in chronic myeloid leukemia. N. Engl. J. Med. 344:1031-1037.

43. Shepard, H.M., Brdlik, C.M., and Schreiber, H. 2008 Signal integration: a framework for understanding the efficacy of therapeutics targeting the human EGFR family. J. Clin. Invest. 118:3574-3581.

44. Sugarman, B.J., et al. 1985. Recombinant human tumor necrosis factor-alpha: effects on proliferation of normal and transformed cells in vitro. Science. 230:943-945.

45. Hudziak, R.M., et al. 1988. Amplified expression of the HER2/ERBB2 oncogene induces resistance to tumor necrosis factor alpha in NIH 3T3 cells. Proc. Natl. Acad. Sci. U. S. A. 85:5102-5106.

46. Hudziak, R.M., et al. 1989. p185HER2 monoclonal antibody has antiproliferative effects in vitro and sensitizes human breast tumor cells to tumor necrosis factor. Mol. Cell. Biol. 9:1165-1172. 